\title{
INVERTIBILITY OF SUBMATRICES OF THE PASCAL MATRIX AND BIRKHOFF INTERPOLATION
}

\section{SCOTT KERSEY}

Department of Mathematical Science

Georgia Southern University

Statesboro, GA 30460-8093

USA

e-mail: scott.kersey@gmail.com

\begin{abstract}
The infinite upper triangular Pascal matrix is $T=\left[\left(\begin{array}{c}j \\ i\end{array}\right)\right]$ for $0 \leq i, j$. It is easy to see that any leading principle square submatrix is triangular with determinant 1 , hence invertible. In this paper, we investigate the invertibility of arbitrary square submatrices $T_{r, c}$ comprised of rows $r=\left[r_{0}, \ldots, r_{m}\right]$ and columns $c=\left[c_{0}, \ldots, c_{m}\right]$ of $T$. We show that $T_{r, c}$ is invertible $r \leq c$ (i.e., $r_{i} \leq c_{i}$ for $i=0, \ldots, m$ ), or equivalently, iff all diagonal entries are nonzero. To prove this result, we establish a connection between the invertibility of these submatrices and polynomial interpolation. In particular, we apply the theory of Birkhoff interpolation and Pölya systems.
\end{abstract}

2010 Mathematics Subject Classification: 15A15, 41A10.

Keywords and phrases: Pascal matrix, Birkhoff interpolation, Pölya system.

Received August 27, 2016

(C) 2016 Scientific Advances Publishers 


\section{Introduction}

The infinite and order $n+1$ upper triangular Pascal matrices are

$$
T:=\left[\left(\begin{array}{l}
j \\
i
\end{array}\right)\right]=\left[\begin{array}{ccccc}
1 & 1 & 1 & 1 & \cdots \\
0 & 1 & 2 & 3 & \cdots \\
0 & 0 & 1 & 3 & \cdots \\
0 & 0 & 0 & 1 & \cdots \\
\vdots & \vdots & \vdots & \vdots & \ddots
\end{array}\right] \text { and } T_{n}:=\left[\begin{array}{ccccc}
1 & 1 & 1 & \cdots & \left(\begin{array}{l}
n \\
0
\end{array}\right) \\
0 & 1 & 2 & \cdots & \left(\begin{array}{l}
n \\
1
\end{array}\right) \\
0 & 0 & 1 & \cdots & \left(\begin{array}{l}
n \\
2
\end{array}\right) \\
\vdots & \vdots & \vdots & \ddots & \vdots \\
0 & 0 & 0 & \cdots & \left(\begin{array}{l}
n \\
n
\end{array}\right)
\end{array}\right] \text {, }
$$

for $i, j=0,1, \ldots$, with $\left(\begin{array}{l}j \\ i\end{array}\right):=0$ if $i>j$. Note that $T_{n}$ is a finite square submatrix of $T$ determined by the first $n+1$ rows and columns. Other (square) submatrices of $T$ are determined by selecting sequences of the rows and columns of $T$. These submatrices can be represented as

$$
T_{r, c}=\left[\left(\begin{array}{c}
c_{j} \\
r_{i}
\end{array}\right)\right]=\left[\begin{array}{cccc}
\left(\begin{array}{c}
c_{0} \\
r_{0}
\end{array}\right) & \left(\begin{array}{c}
c_{1} \\
r_{0}
\end{array}\right) & \ldots & \left(\begin{array}{c}
c_{m} \\
r_{0}
\end{array}\right) \\
\left(\begin{array}{c}
c_{0} \\
r_{1}
\end{array}\right) & \left(\begin{array}{c}
c_{1} \\
r_{1}
\end{array}\right) & \ldots & \left(\begin{array}{c}
c_{m} \\
r_{1}
\end{array}\right) \\
\vdots & \vdots & \ddots & \vdots \\
\left(\begin{array}{c}
c_{0} \\
r_{m}
\end{array}\right) & \left(\begin{array}{c}
c_{1} \\
r_{m}
\end{array}\right) & \ldots & \left(\begin{array}{c}
c_{m} \\
r_{m}
\end{array}\right)
\end{array}\right],
$$

for some selections $r=\left[r_{0}, \ldots, r_{m}\right]$ and $c=\left[c_{0}, \ldots, c_{m}\right]$ of the rows and columns of $T$, respectively.

The main issue considered in this paper is invertibility of these submatrices. It is trivial to see that $T_{n}$ is invertible, with determinant 1. But what about other submatrices? For example,

$$
T_{[0,1,2],[1,2,5]}=\left[\begin{array}{ccc}
1 & 1 & 1 \\
1 & 2 & 5 \\
0 & 1 & 10
\end{array}\right] \text { and } T_{[1,3,4],[1,2,5]}=\left[\begin{array}{ccc}
1 & 2 & 5 \\
0 & 0 & 10 \\
0 & 0 & 5
\end{array}\right] .
$$


The second matrix, a triangular matrix with a zero on the main diagonal, clearly has a zero determinant, hence is not invertible. However, while the first matrix is invertible, it is not obvious to see. In this paper, we will prove the following necessary and sufficient conditions for invertibity of these submatrices:

Theorem 1.1. Submatrices $T_{r, c}$ of the upper Pascal matrix are invertible iff the following equivalent conditions hold:

- $r \leq c\left(\right.$ i.e., $r_{i} \leq c_{i}$ for all $\left.i\right)$.

- There is no zero diagonal entry.

To our knowledge, this result has not appeared in the literature, other than in a preliminary draft of this paper in [4]. The motivation for this work comes from our work on dual basis in subspaces which first appeared in [3]. In that paper, we use the theorem to demonstrate the linear independence of certain dual basis functions.

As it turns out, the problem of invertibility of submatrices of the Pascal matrix is connected to the theory of Birkhoff interpolation. This goes back to a paper of Pölya in [7], as used by Whittaker in [8], and later generalized by Ferguson in [2]. The more general Birkhoff interpolation problem (with more than two interpolation points) was presented by Birkhoff in [1]. In our work, we show that such submatrices of the Pascal matrix are invertible iff a certain 2-point Birkhoff interpolation problem satisfies the Pölya condition.

More recently, in [5] and [6], Birkhoff interpolation is generalized to lacunary interpolation, which could be applied to the problem investigated in this paper. While some of the techniques in their work are similar to ours, we were not aware of their work during the preparation of this paper, and so our results were obtained independently. Finally, while the work in this paper originates to [4], much of the original material has been removed and some content has been changed and/or corrected. In particular, in the original draft we refer to the submatrices as truncations of the Pascal matrix. 
The remainder of this paper is organized as follows:

- In Section 2, we show that our problem is equivalent to a certain two-point polynomial interpolation problem.

- In Section 3, we summarize pertinent aspects of Birkhoff interpolation and Pölya systems.

- In Section 4, we prove Theorem 1.1.

\section{The Pascal Matrix and Polynomial Interpolation}

We begin this paper by establishing a connection between (submatrices of) the Pascal matrix and polynomial interpolation. Let $\Lambda_{\alpha}$ be a row vector

$$
\Lambda_{\alpha}=\left[\frac{\delta_{\alpha}}{0 !}, \frac{\delta_{\alpha} D}{1 !}, \ldots, \frac{\delta_{\alpha} D^{i}}{i !}, \ldots\right]
$$

comprised of the functionals

$$
\delta_{\alpha} D^{i}: f \mapsto f^{(i)}(\alpha)
$$

and let $V$ be the sequence of monomials

$$
V=\left[1,(\cdot),(\cdot)^{2}, \ldots\right] .
$$

Note that $\Lambda_{\alpha}^{T} V$ is an infinite matrix. We will be interested in the maps $\Lambda_{0}$ and $\Lambda_{1}$, when $\alpha=0$ and $\alpha=1$, respectively. In particular, we have the following:

Proposition 2.1. The Pascal matrix $T=\left[\left(\begin{array}{l}j \\ i\end{array}\right)\right]$ is equivalent to

$$
\Lambda_{1}^{T} V=\left[\frac{\delta_{1} D^{i}(\cdot)^{j}}{i !}\right]
$$


Proof. Let $A:=\Lambda_{1}^{T} V$. We will show that $A=T$. For $j \geq i$,

$$
A(i, j)=\frac{\delta_{1} D^{i}}{i !} x^{j}=\left.\frac{1}{i !} \frac{j !}{(j-i) !} x^{j-i}\right|_{x=1}=\left(\begin{array}{l}
j \\
i
\end{array}\right) .
$$

For $j<i$,

$$
A(i, j)=\frac{\delta_{1}}{i !}\left(D^{i} x^{j}\right)=\frac{\delta_{1}}{i !} 0=0
$$

Hence, $A=T$.

We can state a similar result for submatrices of the Pascal matrix. Let $r=\left[r_{0}, \ldots, r_{m}\right]$ and $c=\left[c_{0}, \ldots, c_{m}\right]$ be selections of the rows and and columns of $T$, respectively. Let

$$
\Lambda_{\alpha, r}=\left[\frac{\delta_{\alpha} D^{r_{0}}}{r_{0} !}, \ldots, \frac{\delta_{\alpha} D^{r_{m}}}{r_{m} !}\right],
$$

and

$$
V_{c}=\left[(\cdot)^{c_{0}}, \ldots,(\cdot)^{c_{m}}\right] .
$$

Then, by restricting the result in Proposition 2.1 to the row $r$ and columns $c$, we obtain the following:

Proposition 2.2. Let $r=\left[r_{0}, \ldots, r_{m}\right]$ and $c=\left[c_{0}, \ldots, c_{m}\right]$ be selections of the rows and columns of $T$. Then,

$$
T_{r, c}=\Lambda_{1, r}^{T} V_{c}
$$

Hence, to establish the invertibility of $T_{r, c}$, we can analyze $\Lambda_{1, r}^{T} V_{c}$. To do so, we will establish a connection with two-point polynomial interpolation, which we do by augmenting the point functionals at $x=1$ with functionals at $x=0$. Let $\bar{c}$ be the complement of $c$ in $[0: n]$, with $n:=\max \left\{r_{m}, c_{m}\right\}$. Let

$$
\Lambda_{r, \bar{c}}:=\left[\Lambda_{1, r}, \Lambda_{0, \bar{c}}\right]
$$


and

$$
V_{c, \bar{c}}=\left[V_{c}, V_{\bar{c}}\right]=\left[(\cdot)^{c_{0}}, \ldots,(\cdot)^{c_{d}},(\cdot)^{\bar{c}_{0}}, \ldots,(\cdot)^{\bar{c}_{n-m}}\right] .
$$

Note that $V_{c, \bar{c}}$ is the power basis of degree $n$ with the terms rearranged.

Theorem 2.3. $T_{r, c}$ is invertible iff $\Lambda_{r, \bar{c}}^{T} V_{c, \bar{c}}$ is invertible.

Proof. The interpolation matrix can be expressed in block form as follows:

$$
\Lambda_{r, \bar{c}}^{T} V_{c, \bar{c}}=\left[\Lambda_{1, r}, \Lambda_{0, \bar{c}}\right]^{T}\left[V_{c}, V_{\bar{c}}\right]=\left[\begin{array}{c|c}
\Lambda_{1, r}^{T} V_{c} & \Lambda_{1, r}^{T} V_{\bar{c}} \\
\hline \Lambda_{0, \bar{c}}^{T} V_{c} & \Lambda_{0, \bar{c}}^{T} V_{\bar{c}}
\end{array}\right]
$$

By Proposition 2.2, the upper left block is $T_{r, c}$. The lower left and lower right blocks comprises terms of the form $\frac{1}{i !} \delta_{0} D^{j}(\cdot)^{i}=\left.\frac{1}{i !} D^{j}(\cdot)^{i}\right|_{0}$, which are 1 when $i=j$ and zero otherwise. But since $c$ and $\bar{c}$ are complementary, the lower left block is all zeros, while the lower right block is the identity. Hence,

$$
\Lambda_{r, \bar{c}}^{T} V_{c, \bar{c}}=\left[\begin{array}{c|c}
T_{r, c} & \Lambda_{1, r}^{T} V_{\bar{c}} \\
\hline 0 & I
\end{array}\right] .
$$

Therefore, $\operatorname{det}\left(\Lambda_{r, \bar{c}}^{T} V_{c, \bar{c}}\right)=\operatorname{det}\left(T_{r, c}\right)$, and so $\Lambda_{r, \bar{c}}^{T} V_{c, \bar{c}}$ is invertible iff $T_{r, c}$ is invertible.

For example, suppose $r=[0,2,4,7]$ and $c=[1,2,5,8]$ in $[0: 8]$. Then,

$$
\begin{aligned}
& \bar{c}=[0,3,4,6,7],
\end{aligned}
$$

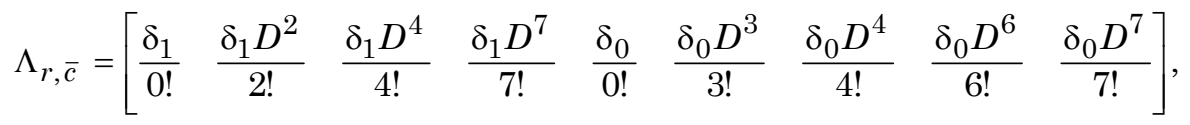

$$
\begin{aligned}
& V_{[c, \bar{c}]}=\left[\begin{array}{lllllllll}
(\cdot) & (\cdot)^{2} & (\cdot)^{4} & (\cdot)^{8} & 1 & (\cdot)^{3} & (\cdot)^{4} & (\cdot)^{6} & (\cdot)^{7}
\end{array}\right] .
\end{aligned}
$$


And so,

$$
\begin{aligned}
& \begin{array}{lllllllll} 
& t^{2} & t^{5} & t^{8} & 1 & t^{3} & t^{4} & t^{6} & t^{7}
\end{array} \\
& \frac{1}{0 !} \delta_{1} \quad\left[\left(\begin{array}{l}
1 \\
0
\end{array}\right)\left(\begin{array}{l}
2 \\
0
\end{array}\right)\left(\begin{array}{l}
5 \\
0
\end{array}\right)\left(\begin{array}{l}
8 \\
0
\end{array}\right) \mid\left(\begin{array}{l}
0 \\
0
\end{array}\right)\left(\begin{array}{l}
3 \\
0
\end{array}\right)\left(\begin{array}{l}
4 \\
0
\end{array}\right)\left(\begin{array}{l}
6 \\
0
\end{array}\right)\left(\begin{array}{l}
7 \\
0
\end{array}\right)\right] \\
& \frac{1}{2 !} \delta_{1} D^{2}\left(\begin{array}{l}
1 \\
2
\end{array}\right)\left(\begin{array}{l}
2 \\
2
\end{array}\right)\left(\begin{array}{l}
5 \\
2
\end{array}\right)\left(\begin{array}{l}
8 \\
2
\end{array}\right)\left(\begin{array}{l}
0 \\
2
\end{array}\right)\left(\begin{array}{l}
3 \\
2
\end{array}\right)\left(\begin{array}{l}
4 \\
2
\end{array}\right)\left(\begin{array}{l}
6 \\
2
\end{array}\right)\left(\begin{array}{l}
7 \\
2
\end{array}\right) \\
& \frac{1}{4 !} \delta_{1} D^{4}\left(\begin{array}{l}
1 \\
4
\end{array}\right)\left(\begin{array}{l}
2 \\
4
\end{array}\right)\left(\begin{array}{l}
5 \\
4
\end{array}\right)\left(\begin{array}{l}
8 \\
4
\end{array}\right)\left(\begin{array}{l}
0 \\
4
\end{array}\right)\left(\begin{array}{l}
3 \\
4
\end{array}\right)\left(\begin{array}{l}
4 \\
4
\end{array}\right)\left(\begin{array}{l}
6 \\
4
\end{array}\right)\left(\begin{array}{l}
7 \\
4
\end{array}\right) \\
& \begin{array}{l}
\frac{1}{7 !} \delta_{1} D^{7} \quad\left(\begin{array}{l}
1 \\
7
\end{array}\right)\left(\begin{array}{l}
2 \\
7
\end{array}\right)\left(\begin{array}{l}
5 \\
7
\end{array}\right)\left(\begin{array}{l}
8 \\
7
\end{array}\right)\left(\left(\begin{array}{l}
0 \\
7
\end{array}\right)\left(\begin{array}{l}
3 \\
7
\end{array}\right)\left(\begin{array}{l}
4 \\
7
\end{array}\right)\left(\begin{array}{l}
6 \\
7
\end{array}\right)\left(\begin{array}{l}
7 \\
7
\end{array}\right)\right. \\
\hline
\end{array} \\
& \Lambda_{r, \bar{c}}^{T} V_{c, \bar{c}}=\frac{1}{0 !} \delta_{0} \quad \begin{array}{lllll|lllll}
0 & 0 & 0 & 0 & 1 & 0 & 0 & 0 & 0
\end{array} \text {. } \\
& \frac{1}{3 !} \delta_{0} D^{3} \mid \begin{array}{lllll|lllll}
0 & 0 & 0 & 0 & 0 & 1 & 0 & 0 & 0
\end{array} \\
& \frac{1}{4 !} \delta_{0} D^{4} \quad 0 \quad 0000000000
\end{aligned}
$$

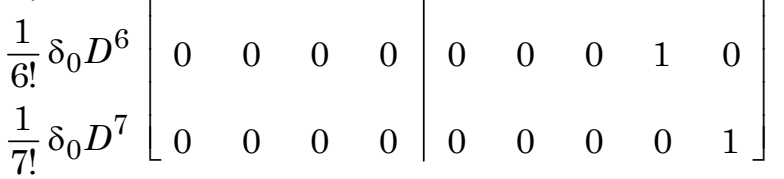

\section{Birkhoff Interpolation and the Pölya Condition}

In the previous section, we established an equivalent condition for the invertibility of the submatrix $T_{r, \bar{c}}$ of the Pascal matrix. This equivalent condition was expressed in terms of a kind of generalized Vandermonde determined by the evaluation of certain derivatives at 0 and 1. As it turns out, this kind of interpolation problem is called (2point) Birkhoff interpolation. To solve problems like this, Ferguson ([2]) used incidence matrices.

Definition 3.1. An incidence matrix $E$ for 2-point interpolation problems on $\prod_{n}$ is a $2 \times(n+1)$ matrix

$$
E:=\left[e_{i j}\right]=\left[\begin{array}{llll}
e_{00} & e_{01} & \cdots & e_{0 m} \\
e_{10} & e_{11} & \cdots & e_{1 m}
\end{array}\right]
$$

of ones and zeros, with exactly $n+1$ ones. The term $e_{i j}$ is 1 when the interpolation problem includes $\delta_{x_{i}} D^{j}$. 
For example, the following are incidence matrices, each of dimension $2 \times 6$ with exactly 6 ones:

$$
\left[\begin{array}{llllll}
0 & 1 & 0 & 1 & 0 & 0 \\
1 & 0 & 1 & 1 & 0 & 1
\end{array}\right], \quad\left[\begin{array}{llllll}
0 & 1 & 0 & 0 & 1 & 1 \\
0 & 1 & 1 & 0 & 1 & 0
\end{array}\right] .
$$

These correspond to the functionals

$$
\left[\delta_{x_{0}}, \delta_{x_{0}}^{3}, \delta_{x_{1}}, \delta_{x_{1}}^{2}, \delta_{x_{1}}^{3}, \delta_{x_{1}}^{5}\right]
$$

and

$$
\left[\delta_{x_{0}}, \delta_{x_{0}}^{4}, \delta_{x_{0}}^{5}, \delta_{x_{1}}, \delta_{x_{1}}^{2}, \delta_{x_{1}}^{4}\right]
$$

respectively. In this paper, we take $x_{0}=0$ and $x_{1}=1$. As in [2], we define $M_{j}$ to be the cumulative column sum

$$
M_{j}:=\sum_{i=0}^{1} \sum_{k=0}^{j} e_{i k}
$$

for $j=0: n$. In the previous examples, $M=[1,2,3,5,5,6]$ and $M=[0,2,3,3,5,6]$. Note that $M_{5}=6$ is the total number of ones in both examples. In general, $M_{n}=n+1$ for any incidence matrix.

The problem of 2-point Birkhoff interpolation was studied by Pölya. With respect to the incidence matrices, the following defintion is used:

Definition 3.2 ([2]). The incidence matrix $E$ satisfies the Pölya condition if $M_{j}>j$ for $j=0: m$.

In the above two examples, the first matrix is Pölya, however the second is not because $M_{0}=0<1$ and $M_{3}=3<4$. The following result, proved independently by Pölya and Whittaker (as also described in [2]), gives necessary and sufficient conditions for correct interpolation. 
Theorem 3.3 (Adapted from [7], [8]). Let $E$ be a $2 \times(n+1)$ incidence matrix with entries $e_{i j}$, ones or zeros. Let

$$
\Lambda:=\left[\delta_{x_{i}} D^{j}: e_{i j}=1\right]
$$

and $V^{n}=\left[1,(\cdot), \ldots,(\cdot)^{n}\right]$. Then, the system $\Lambda^{T} V^{n}$ is invertible iff $E$ satisfies the Pölya condition.

\section{Invertibility of Submatrices of the Pascal Matrix}

Suppose $r=\left[r_{0}, \ldots, r_{m}\right]$ and $c=\left[c_{0}, \ldots, c_{m}\right]$ are selections (increasing sequences) of the rows and columns of $T$, respectively. Let $\bar{c}$ be the complement of $c$ in $[0: n]$ with $n:=\max \left\{r_{m}, c_{m}\right\}$. We define $E_{r, \bar{c}}$ to be the $2 \times(n+1)$ matrix with $E_{r, \bar{c}}(0, j)=1$ if $r_{i}=1$, and $E_{r, \bar{c}}(1, j)=1$ if $\bar{c}_{i}=1$, with all other entries 0 . For example, let $r=[0,1,4]$ and $c=[0,4,5]$. We choose $n=5$. Then, $\bar{c}=[1,2,3]$ and

$$
E_{r, \bar{c}}=E_{[0,1,4],[1,2,3]}=\left[\begin{array}{cccccc}
1 & 1 & 0 & 0 & 1 & 0 \\
0 & 1 & 1 & 1 & 0 & 0
\end{array}\right] .
$$

The following verifies that $E_{r, \bar{c}}$ is an incidence matrix.

Lemma 4.1. $E_{r, \bar{c}}$ is an incidence matrix of dimension $2 \times(n+1)$.

Proof. Since,

$$
\# \bar{c}=(n+1)-\# c=(n+1)-(m+1)=n-m,
$$

it follows that

$$
\sum_{i=0}^{1} \sum_{j=0}^{n} e_{i j}=\# r+\# \bar{c}=(m+1)+(n-m)=n+1 .
$$

Hence, there are exactly $n+1$ ones (and $n+1$ zeros). Following Definition 3.1, $E_{r, \bar{c}}$ is an incidence matrix. 
The next results are used to prove Theorem 1.1. The first is a corollary of two previous theorems.

Corollary 4.2. The Pascal submatrix $T_{r, c}$ is invertible iff $E_{r, \bar{c}}$ is Pölya.

Proof. By Theorem 2.3, $T_{r, c}$ is invertible iff $\Lambda_{r, \bar{c}}^{T} V_{c, \bar{c}}$ is invertible, and by Theorem 3.3, $\Lambda_{r, \bar{c}}^{T} V_{c, \bar{c}}$ is invertible iff $E_{r, \bar{c}}$ is Pölya. Therefore, $T_{r, c}$ is invertible iff $E_{r, \bar{c}}$ is Pölya.

Lemma 4.3. $r \leq c$ iff $\bar{c} \leq \bar{r}$.

Proof. Suppose $r \leq c$. By the tautologies

$$
\begin{aligned}
& \bar{r}-\bar{c}=\bar{r} \cap c=c \cap \overline{r \cap c}, \\
& \bar{c}-\bar{r}=\bar{c} \cap r=r \cap \overline{r \cap c},
\end{aligned}
$$

we see that $\bar{r}$ contains those elements in $c$ that are not in both $c$ and $r$, and $\bar{c}$ contains those elements in $r$ that are not in both $c$ and $r$. Since $r \leq c$, it follows that $\bar{r} \geq \bar{c}$.

For the reverse implication, assume that $\bar{c} \leq \bar{r}$, and apply a similar proof as above, noting that $\overline{\bar{r}}=r$ and $\overline{\bar{c}}=c$.

Lemma 4.4. Let $r$ and $x$ be selection vectors of the same length. Then, $E_{r, x}$ is Pölya iff $x \leq \bar{r}$.

Proof. Suppose first that $x=\bar{r}$. We want to establish that $E_{r, \bar{r}}$ is Pölya. Since $r$ and $\bar{r}$ are complementary, each column of the incidence matrix $E_{r, \bar{r}}$ has exactly one "1" and one "0". Hence, $M_{0}=1, M_{1}=1+1=2$, etc. In particular, $M_{j}=j+1$ for $j=0, \ldots, n$, implying that $E_{r, \bar{r}}$ is Pölya. 
Now, suppose that $x \leq \bar{r}$. Let $M^{x}:=\left[M_{0}^{x}, \ldots, M_{n}^{x}\right]$ and $M^{\bar{r}}:=$ $\left[M_{0}^{\bar{r}}, \ldots, M_{n}^{\bar{r}}\right]$ be the cumulative sums defined in (1). Since $x \leq \bar{r}$, it follows that

$$
M_{j}^{x} \geq M_{j}^{\bar{r}}=j+1
$$

Hence, $E_{r, x}$ is Pölya.

Now suppose that $x \leq \bar{r}$. Then, $x_{i}>\bar{r}_{i}$ for some $i$. Suppose that $j$ is the first such occurrence. Then, $M_{j-1}^{x}=j=M_{j}^{x}$. Hence, we don't have $M_{j}^{x}>j$ for all $j$, implying that $E_{r, x}$ is not Pölya. By contraposition, $E_{r, x}$ Pölya implies $x \leq \bar{r}$.

On combining these last two results, we have $E_{r, x}$ Pölya iff $x \leq \bar{r}$.

We now establish our main result.

Theorem 1.1. Submatrices $T_{r, c}$ of the upper Pascal matrix are invertible iff the following equivalent conditions hold:

- $r \leq c\left(\right.$ i.e., $r_{i} \leq c_{i}$ for all $\left.i\right)$.

- There is no zero diagonal entry.

Proof. For the first part, $r \leq c$ iff $\bar{c} \leq \bar{r}$ by Lemma 4.3, and $\bar{c} \leq \bar{r}$ iff $E_{r, \bar{c}}$ is Pölya by Lemma 4.4. Hence, $E_{r, \bar{c}}$ is Pölya iff $r \leq c$. By Corollary 4.2, this holds iff $T_{r, c}$ is invertible. This establishes the first part.

For the second part, note that the diagonal elements of $T_{r, c}$ are of the form $\left(\begin{array}{l}c_{k} \\ r_{k}\end{array}\right)$, which are zero iff $r_{k}>c_{k}$. Hence, a diagonal element is zero iff $r \not c$. By the first result, this occurs iff $T_{r, c}$ is not invertible. By contraposition, $T_{r, c}$ is invertible iff there is no zero on the diagonal. 


\section{References}

[1] G. D. Birkhoff, General mean value and remainder theorems with applications to mechanical differentiation and integration, Trans. Amer. Math. Soc. 1 (1906), 107-136.

[2] D. Ferguson, The question of uniqueness for G. D. Birkhoff interpolation problems, J. Approx. Th. 2 (1969), 1-28.

[3] S. Kersey, Dual basis functions in subspaces of inner product spaces, Applied Mathematics and Computation 219 (2013), 10012-10024.

[4] S. Kersey, Invertibility of Submatrices of Pascal's Matrix and Birkhoff Interpolation (preprint). ArXiv:1303.6159, 2013.

[5] F. Palacios-Quiñonero, P. Rubió-Díaz, J. Díaz-Barrero and J. Rossell, Order regularity of two-node Birkhoff interpolation with lacunary polynomials, Applied Mathematics Letters 22 (2009), 386-389.

[6] F. Palacios-Quiñonero, P. Rubió-Díaz, J. Díaz-Barrero and J. Rossell, Order of regularity for Birkhoff interpolation with lacunary polynomials, Mathematica Aeterna 1(3) (2011), 129-135.

[7] G. Pölya, Bemerkungen zur Interpolation und zur Näherungstheorie der Balkenbiegung, Z. Angew. Math. Mech. 11 (1931), 4445-449.

[8] J. M. Whittaker, Interpolatory Function Theory, Cambridge University Press, London, 1935. 